\title{
Contenidos De Celulosa Y Lignina En Restos Lignino- Celulósicos De Gran Tamaño (Necromasa) En Un Bosque Templado De Antiguo Crecimiento Del Centro-Sur de Chile
}

\section{Carlos Belezaca Pinargote}

Universidad Técnica Estatal de Quevedo, Facultad de Ciencias Ambientales. Quevedo, Ecuador

\author{
Roberto Godoy Bórquez
}

Universidad Austral de Chile, Facultad de Ciencias. Valdivia, Chile

Darwin Salvatierra Pilozo,

María Cadme Arévalo,

Universidad Técnica Estatal de Quevedo,

Facultad de Ciencias Ambientales. Quevedo, Ecuador

Eduardo Valenzuela Flores

Universidad Austral de Chile, Facultad de Ciencias. Valdivia, Chile

Rolando López Tobar

Diana Delgado Campusano

Renato Baque Mite

Roberto Herrera Anangonó

Universidad Técnica Estatal de Quevedo,

Facultad de Ciencias Ambientales. Quevedo, Ecuador

Carmen Vásconez Montufar

Tito Bohórquez Barros

Universidad Técnica de Babahoyo,

Carrera de Ingeniería Agropecuaria. Babahoyo, Ecuador

doi: 10.19044/esj.2016.v12n24p403 URL:http://dx.doi.org/10.19044/esj.2016.v12n24p403

\begin{abstract}
The evaluation of volume, necromass, states of decay, solubility, and cellulose and lignin content in coarse woody debris (RLC) accumulated in an evergreen temperate forest of old-growth in the Puyehue National Park, South-Central Chile. Ten plots of $900 \mathrm{~m}^{2}$ each, were quantified for RLC $(\geq$ $10 \mathrm{~cm}$ diameter). For necromass quantification was used a scale of five categories/states of decay, necromass $(1=$ lowest and $5=$ highest
\end{abstract}


degradation). $632 \mathrm{~m}^{3} \mathrm{ha}^{-1}$ of deadwood (= 231,5 $\mathrm{Mg} \mathrm{ha}^{-1}$ of necromass) was found, mainly represented by Nothofagus betuloides (95,2\%). The wood with the most advanced state of decay (state 5) showed a greater solubility, increasing of 273,7 and 818,6 times more soluble than 1, for $N$. betuloides and $S$. conspicua, respectively. Cellulose content decreased to $91 \%$, while the lignin increased $248 \%$ and $142 \%$ in wood decay of $N$. betuloides and $S$. conspicua, respectively. These results demonstrate the importance of RLC in the biogeochemistry of remote forest ecosystems of old-growth in Southern Chile.

Keywords: Biogeochemical, decaying wood, dead wood, wood solubility, logs.

\section{Resumen}

Se evaluó el volumen, necromasa, estados de descomposición, solubilidad, y contenidos de celulosa y lignina en restos lignino-celulósicos de gran tamaño (RLC) acumulados en un bosque templado siempreverde de antiguo crecimiento dentro del Parque Nacional Puyehue, Centro-Sur de Chile. En 10 parcelas de $900 \mathrm{~m}^{2}$, se cuantificó los RLC ( $\left.\geq 10 \mathrm{~cm} \phi\right)$, cuya necromasa se clasificó empleando una escala de cinco categorías/estados de descomposición (1= menor degradación y 5= mayor degradación). Se encontró $632 \mathrm{~m}^{3} \mathrm{ha}^{-1}$ de madera muerta (= 231,5 $\mathrm{Mg} \mathrm{ha}^{-1}$ de necromasa), representados principalmente por Nothofagus betuloides (95,2\%). La madera en el estado más avanzado de degradación (estado 5) presentó mayor solubilidad, con un aumento de 273,7 y 818,6 veces más soluble que el estado 1 , para $N$. betuloides y $S$. conspicua, respectivamente. La celulosa disminuyó hasta 91\%, mientras la lignina aumentó 248\% y 142\% en madera degradada de $N$. betuloides y $S$. conspicua, respectivamente. Estos resultados demuestran la importancia de los RLC en la biogeoquímica de ecosistemas remotos boscosos de antiguo crecimiento del sur de Chile.

Palabras clave: Biogeoquímica, descomposición de madera, madera muerta, solubilidad de madera, troncos.

\section{INTRODUCCIÓN}

La acumulación de litera leñosa muerta, formada por RLC (troncos, tocones, raíces, ramas, etc.) sobre y bajo el piso forestal, ocurre como producto de disturbios naturales (bosques prístinos) y antropogénicos (bosques intervenidos), (Carmona et al., 2002; Schlegel \& Donoso, 2008). Bajo condiciones prístinas, los RLC se acumulan en el suelo, cuando el bosque sufre disturbios naturales recurrentes, como actividad volcánica, 
vientos, nevadas, lluvias torrenciales, derrumbes, o en su defecto ataques de plagas, especialmente en árboles que han llegado al final de su ciclo biológico (Brown et al., 2013), y su presencia es evidente, constituyendo un componente funcional y estructural como hábitat para productores y consumidores (Jia-bing et al., 2005) que tributan a la biogeoquímica del sistema (Heinemann \& Kitzberger, 2006).

En bosques templados maduros y de lento crecimiento del sur de Chile, se ha estimado que entre el $86 \%$ y $94 \%$ del total de RLC que constituyen la necromasa gruesa (Schlegel \& Donoso, 2008) observada en el presente, pertenecen a árboles muertos hace décadas (Lombardi et al., 2011), y su relevancia es evidente en las diferentes etapas de desarrollo del bosque (Césari et al., 2010). Entre las contribuciones de esta necromasa, se destacan la función de sustrato para plántulas de regeneración del bosque, hábitat para meso y microbiota, etc., (Brown et al., 2013). Durante su descomposición se liberan gradualmente nutrientes y formas orgánicas e inorgánicas de carbono (C), (Watkinson et al., 2006), constituyendo uno de los procesos más importantes del reciclaje en ecosistemas terrestres, por el aporte de nutrientes al suelo y la formación de materia orgánica (González-Polo et al., 2013).

La presencia de RLC en ecosistemas boscosos prístinos del sur de Chile, y estudios a nivel de campo dan cuenta que estos componentes orgánicos se encuentran en diferentes etapas de degradación (Belezaca et al., 2016), mineralizándose lentamente, contribuyendo a los procesos biogeoquímicos del ecosistema (Lombardi et al., 2011). Los procesos degradativos conllevan pérdidas y transformación bioquímica de componentes, con los consecuentes cambios estructurales en la madera.

En función a lo anteriormente mencionado, en esta investigación se planteó estudiar la solubilidad y contenidos de celulosa y lignina en restos lignino-celulósicos acumulados sobre el suelo de un bosque templado siempreverde de antiguo crecimiento del Centro-Sur de Chile.

\section{MÉTODOS}

Área de estudio. Estuvo ubicada en la Cordillera de Los Andes, en una formación vegetal de bosque templado siempreverde de antiguo crecimiento, dentro del Parque Nacional Puyehue, Centro-Sur de Chile $\left(40^{\circ}\right.$ 47’ S - 72 $12^{\prime} \mathrm{O}$ ), donde Nothofagus betuloides (Mirb.) Oerst (coigue) fue la especie forestal que dominó el estrato superior, seguido de Saxegothaea conspicua Lindl. (mañio hembra) y Laureliopsis philippiana (Looser) R. Schodde (tepa) que predominaron en el estrato medio del bosque, con edades superiores a los 300 años. En el estrato arbustivo bajo ( $<4 \mathrm{~m}$ de altura) prevalecieron especies del género Chusquea. El suelo es de topografía irregular, y se originó a partir de cenizas volcánicas Andesítico-Basálticas, depositadas sobre diferentes sustratos, clasificándose como suelos Andisoles 
(Typic Dystrandepts) de acuerdo al Soil Survey Staff (1999). El clima es lluvioso con una precipitación promedio, superior a $7000 \mathrm{~mm}$ anuales y una temperatura media anual del aire de $4,5^{\circ} \mathrm{C}$ (Oyarzún et al., 2004).

Establecimiento de parcelas, estimación del volumen y necromasa lignino-celulósica. Durante noviembre del año 2013, se establecieron 10 parcelas de $900 \mathrm{~m}^{2}(30 \mathrm{x} 30 \mathrm{~m})$ en un área de bosque dominado por $N$. betuloides, y otras especies forestales significativas, entre las que sobresalen Saxegothaea conspicua Lindl. (mañio hembra) y Laureliopsis philippiana (Looser) R. Schodde (tepa), con edades superiores a los 300 años. Se midió el diámetro (mayor, medio, y menor), y longitud de los restos lignino-celulósicos gruesos $(\geq 10 \mathrm{~cm}$ de diámetro), e identificó la(s) especie(s) forestal(es) a quien pertenecían. Posteriormente, los datos recopilados se procesaron, analizaron y mediante la fórmula de Smaliam [1] sugerida para cubicar trozas apeadas, se estimó el volumen de RLC.

$$
\begin{aligned}
& \mathrm{V}=\left(\frac{\pi}{4}\right) *\left(\frac{D_{\text {mayor }+} D_{\text {medio }+} D_{\text {menor }}}{3}\right)^{2} * L \\
& \text { Donde: } \mathrm{V}=\text { Volumen }\left(\mathrm{m}^{3}\right) \text {. } \\
& \pi=3,1416 \text {. } \\
& \mathrm{D}=\text { Diámetro }(\mathrm{m}) \text {. } \\
& \mathrm{L}=\text { Longitud }(\mathrm{m}) \text {. }
\end{aligned}
$$

Los RLC gruesos se clasificados en 5 categorías, de acuerdo a sus características físicas y grado de descomposición, siguiendo los criterios propuestos por Spies et al. (1988), Marra y Edmonds (1994), Yan et al. (2006), (Cuadro 1).

Cuadro 1. Clasificación de RLC gruesos de acuerdo a los criterios sugeridos por Spies et al. (1988), Marra y Edmonds (1994), y Yan et al. (2006).

\begin{tabular}{clll}
\hline CATEGORÍAS & \multicolumn{4}{c}{ CARACTERÍSTICAS } \\
\hline 1 & Material recientemente caído con tejidos y corteza intactos. & \\
\hline 2 & $\begin{array}{l}\text { Albura empezando a descomponerse, pero completamente presente. Corteza } \\
\text { empezando a romperse. }\end{array}$ \\
\hline 3 & Albura y corteza mayormente presente, duramen intacto. & \\
\hline 4 & Corteza y albura casi desaparecida, duramen empezando a descomponerse. & \\
\hline 5 & $\begin{array}{l}\text { Albura y corteza desaparecido, duramen con avanzado estado de } \\
\text { descomposición. }\end{array}$
\end{tabular}

La biomasa seca se determinó multiplicando el volumen de cada sección de madera, por la densidad específica $\left(\mathrm{g}^{-1} \mathrm{~cm}^{-3}\right)$, como lo realizó Gayoso (2001) en especies forestales de bosques siempreverdes de Chile, similares a las del presente estudio. Se utilizó los valores de densidad 0,51; 0,$44 ; 0,36 ; 0,31 ;$ y $0,25 \mathrm{~g}^{-1} \mathrm{~cm}^{3}$ asignados a cada una de las cinco categorías de descomposición, respectivamente. 
Solubilidad de madera en $\mathrm{NaOH} 1 \%$. Tres muestras de madera (tres repeticiones), de $N$. betuloides (latifoliada) y $S$. conspicua (conífera nativa), correspondientes a cada categoría de descomposición se secaron en una estufa a $70{ }^{\circ} \mathrm{C}$ durante 8 días. La solubilidad se determinó siguiendo la metodología propuesta en TAPPI 212 om-98. Brevemente, 2 g de aserrín seco al aire, de humedad conocida y tamizado entre mallas de 40 - 60 mesh $(0,40-0,25 \mathrm{~mm})$, con sus extraíbles intactos, se depositaron en un Erlenmeyer, al que se agregó $100 \mathrm{~mL}$ de una solución de $\mathrm{NaOH} 1 \%$, e introducidos en un baño termoregulado $\left(97{ }^{\circ} \mathrm{C}-100{ }^{\circ} \mathrm{C}\right)$ durante 60 minutos, para posteriormente la mezcla ser enfriada y filtrada. Finalmente, el filtro con aserrín atrapado se secó y pesó. El porcentaje de madera soluble se calculó mediante la fórmula [2].

$$
\begin{aligned}
& \text { \% de solubilidad }=\frac{(A-B)}{A} * 100 \\
& \begin{aligned}
\text { Donde: } & A=\text { Peso seco de la muestra antes de la extracción (g). } \\
B & =\text { Peso seco de la muestra después de la extracción (g). }
\end{aligned}
\end{aligned}
$$

Análisis de celulosa y lignina. Similar al caso anterior, tres muestras de necromasa lignino-celulósica (tres repeticiones), de $N$. betuloides y $S$. conspicua, correspondientes a cada categoría de descomposición se secaron en una estufa a $70{ }^{\circ} \mathrm{C}$ durante 8 días, hasta peso constante. La determinación de celulosa se realizó mediante el método de Kurschner \& Hoffer, usado por Crespo et al. (2013). Dicho método consistió en depositar 1 g de aserrín de madera seca (molido a 40 - 60 mesh), libre de extraíbles (TAPPI 204 cm-97) en un Erlenmeyer y mezclar con $20 \mathrm{~mL}$ de etanol y $5 \mathrm{~mL}$ de ácido nítrico concentrado. La mezcla se hirvió a reflujo durante 30 minutos, dejó enfriar y decantar, para finalmente eliminar el líquido por filtración. Esta operación se realizó en tres oportunidades. El filtro con la celulosa retenida se secó y pesó. El porcentaje de celulosa para cada uno de los cinco estados de degradación se calculó según la siguiente ecuación [3].

$$
\begin{aligned}
& \%=\frac{A \times 100}{W} \\
& \text { Donde: } \mathrm{A}=\text { Peso del residuo seco ( } \mathrm{g} \text { ). } \\
& \mathrm{W}=\text { Peso seco de la muestra }
\end{aligned}
$$

Para el caso de la lignina, se empleó la metodología propuesta en las normas TAPPI (TAPPI 222 om-98), que determina lignina insoluble en ácido, o también llamada lignina Klason. Brevemente, 1 g de aserrín seco al aire, libre de extraíbles (TAPPI 204 cm-97), de humedad conocida y tamizado entre mallas de $40-60$ mesh $(0.40-0.25 \mathrm{~mm})$, se depositó en un 
Erlenmeyer y maceró con $\mathrm{H}_{2} \mathrm{SO}_{4}$ al $72 \%$ durante 2 horas a $20 \pm 1{ }^{\circ} \mathrm{C}$. Luego, se agregó agua suficiente hasta reducir la concentración del ácido al 3\%, e hirvió a reflujo durante 4 horas. Pasado este tiempo, la mezcla se enfrió, decantó y luego filtró. El filtro con el producto retenido se secó y pesó. Para los cálculos del porcentaje de lignina en cada uno de los cinco estados de degradación de la madera, se utilizó la formula [3].

Análisis estadísticos. Los datos obtenidos por cada variable estudiada, se sometieron a un análisis de varianza (ANOVA) con un nivel de significancia de 95\% $(P<0.05)$, previa comprobación de los supuestos de normalidad y homocedasticidad de varianzas. Posteriormente se aplicó la prueba LSD (mínima diferencia significativa), con un nivel de significancia del 95\% $(P<0.05)$. Todos los análisis estadísticos se efectuaron en el programa STATISTICA 7, versión para Windows.

\section{RESULTADOS}

Caracterización cuali-cuantitativa de RLC. La mayor parte perteneció principalmente a $N$. betuloides con $602\left(\mathrm{~m}^{3} \mathrm{ha}^{-1}\right)(95 \%), S$. conspicua $(3,7 \%)$ y en menor cantidad a L. phillipiana $(0,8 \%)$ y Dasyphyllum diacanthoides (Less.) Cabrera (0,3\%). En el cuadro 2 se muestran los aportes $\left(\mathrm{m}^{3} \mathrm{ha}^{-1}\right)$ y representatividad (\%) de RLC.

Cuadro 2. Volumen $\left(\mathrm{m}^{3} \mathrm{ha}^{-1}\right)$ y representatividad (\%) de restos ligninocelulósicos depositados en un bosque templado de antiguo crecimiento dominado por Nothofagus, Parque Nacional Puyehue, Centro-Sur de Chile. Valores corresponden a promedios de 10 parcelas con su respectivo error estándar.

\begin{tabular}{lcccc}
\hline \multicolumn{1}{c}{ Variables } & $\begin{array}{c}\text { Nothofagus } \\
\text { betuloides }\end{array}$ & $\begin{array}{c}\text { Saxegothaea } \\
\text { conspicua }\end{array}$ & $\begin{array}{c}\text { Laureliopsis } \\
\text { philippiana }\end{array}$ & $\begin{array}{c}\text { Dasyphyllum } \\
\text { diacanthoides }\end{array}$ \\
\hline Volumen $\left(\mathrm{m}^{3} \mathrm{ha}^{-1}\right)$ & $602( \pm 100,6)$ & $23,4( \pm 5,4)$ & $5,1( \pm 3,4)$ & $1,9( \pm 1,7)$ \\
\hline Representatividad $(\%)$ & 95,2 & 3,7 & 0,8 & 0,3 \\
\hline
\end{tabular}

La mayor parte de RLC se encontraron en los estados de descomposición 4 y 5 (según escala), con 218 y 126 unidades por ha ${ }^{-1}$ como promedio, representando el $57,7 \%$ y $33 \%$, respectivamente, mientras que la presencia de restos leñosos en las categorías 1,2 y 3 , fue de baja representatividad. La totalidad de RLC constituyeron $632 \mathrm{~m}^{3} \mathrm{ha}^{-1}$ de volumen, que representaron 231,5 $\mathrm{Mg}^{-1} \mathrm{ha}^{-1}$ de necromasa, encontrada principalmente en estados avanzados de descomposición (escala 4 y 5), (cuadro 3). 
Cuadro 3. Número, porcentaje, volumen $\left(\mathrm{m}^{3} \mathrm{ha}^{-1}\right)$ y necromasa $\left(\mathrm{Mg} \mathrm{ha}^{-1}\right)$ de RLC en cinco estados de descomposición, según Spies et al. (1988), Marra y Edmonds (1994), y Yan et al. (2006), encontrados en un bosque templado de antiguo crecimiento dominado por Nothofagus, Parque Nacional Puyehue, Centro-Sur de Chile. Valores corresponden a promedios de 10 parcelas con su respectivo error estándar.

\begin{tabular}{lccccc}
\multirow{2}{*}{ Variables } & \multicolumn{5}{c}{ Estados de descomposición según escala } \\
\cline { 2 - 6 } & $\mathbf{1}$ & $\mathbf{2}$ & $\mathbf{3}$ & $\mathbf{4}$ & $\mathbf{5}$ \\
\hline Número de RLC & $8( \pm 3,9)$ & $7( \pm 2,4)$ & $208( \pm 8,2)$ & $218( \pm 25)$ & $126( \pm 14)$ \\
\hline Representatividad $(\%)$ & 2,4 & 1,6 & 5,3 & 57,7 & 33 \\
\hline Volumen $\left(\mathrm{m}^{3} \mathrm{ha}^{-1}\right)$ & $2,22( \pm 1,3)$ & $0,83( \pm 0,5)$ & $71,26( \pm 43,2)$ & $375,74( \pm 74,6)$ & $181,74( \pm 41,8)$ \\
\hline Necromasa $\left(\mathrm{Mg}^{-1} \mathrm{ha}^{-1}\right)$ & $1,25( \pm 0,7)$ & $0,44( \pm 0,2)$ & $34,21( \pm 20,7)$ & $146,54( \pm 29,1)$ & $49,07( \pm 11,3)$ \\
\hline
\end{tabular}

Solubilidad de madera en $\mathrm{NaOH} 1 \%$. Se encontraron diferencias estadísticas significativas $(P<0,001)$ entre los cuatro primeros estados de descomposición y el quinto estado de descomposición de la madera de $N$. betuloides y $S$. conspicua. La madera en avanzado estado de degradación (estado 5) presentó mayor solubilidad, donde $N$. betuloides alcanzó el 34,9\%, y $S$. conspicua el $48,9 \%$, representando un incremento en la solubilidad de la madera de 273,71 y 818,61 veces, entre el estado de descomposición 1 y el 5. Mientras que los estados $1-4$ tuvieron baja solubilidad (cuadro 4).

Cuadro 4. Solubilidad de madera (\%) de $N$. betuloides y $S$. conspicua en $\mathrm{NaOH} 1 \%$, bajo cinco estados de descomposición. Parque Nacional Puyehue, Centro-Sur de Chile.

\begin{tabular}{ccccc}
\hline \multirow{2}{*}{ ED } & \multicolumn{5}{c}{ SOLUBILIDAD DE MADERA EN NaOH 1\% } \\
\cline { 2 - 5 } & \multicolumn{3}{c}{$\boldsymbol{N}$. betuloides } & \multicolumn{3}{c}{ S. conspicua } \\
\hline 1 & $9,36( \pm 0,26)$ a & $---{ }^{\mathrm{A}}$ & $5,32( \pm 0,13)$ a & $-{ }^{---{ }^{\mathrm{B}}}$ \\
\hline 2 & $9,52( \pm 0,11)$ a & 1,71 & $6,01( \pm 0,11)$ a & 12,97 \\
\hline 3 & $10,40( \pm 0,02)$ a & 11,11 & $8,01( \pm 0,17)$ a & 50,56 \\
\hline 4 & $10,87( \pm 0,36)$ a & 16,13 & $14,99( \pm 1,51)$ a & 181,77 \\
\hline 5 & $34,98( \pm 9,46)$ b & 273,71 & $48,87( \pm 1,30)$ b & 818,61 \\
\hline
\end{tabular}

Valores corresponden a promedios de tres repeticiones con su respectiva desviación estándar. Letras distintas indican medias estadísticamente diferentes $(P<0,05)$.

ED: Estados de descomposición según escala sugerida por Spies et al. (1988), Marra y Edmonds (1994), y Yan et al. (2006).

${ }^{\mathbf{A}} \mathrm{y}^{\mathbf{B}}$ : Incrementos (\%) en la solubilidad de madera de $N$. betuloides y $S$. conspicua con respecto al estado inicial de degradación.

Contenidos de celulosa y lignina. Los estados iniciales de descomposición, presentaron mayores contenidos (\%) de celulosa, no obstante, conforme aumentó la degradación, los contenidos decrecieron gradualmente hasta un máximo de 91\%, en el estado de degradación 5. Por el contrario, la concentración de lignina aumentó gradualmente en madera más 
degradada, llegando a ser 248\% y 142\% mayor para madera en estado de degradación 5, respecto al estado inicial de descomposición, en $N$. betuloides y $S$. conspicua, respectivamente (cuadro 5). Estos resultados permitieron detectar diferencias estadísticas significativas $(P<0.001)$ para ambas variables analizadas, entre los cinco estados de descomposición para la necromasa de $N$. betuloides y $S$. conspicua.

Cuadro 5. Contenidos (\%) de celulosa y lignina en necromasa ligninocelulósica de $N$. betuloides y $S$. conspicua bajo cinco estados de descomposición (ED). Parque Nacional Puyehue, Centro-Sur de Chile.

\begin{tabular}{|c|c|c|c|c|c|c|c|c|}
\hline \multirow{2}{*}{ ED } & \multicolumn{4}{|c|}{ CELULOSA } & \multicolumn{4}{|c|}{ LIGNINA } \\
\hline & \multicolumn{2}{|c|}{ N. betuloides } & \multicolumn{2}{|c|}{ S. conspicua } & \multicolumn{2}{|c|}{ N. betuloides } & \multicolumn{2}{|c|}{ S. conspicua } \\
\hline 1 & $47.91( \pm 1.15) \mathrm{a}$ & $\cdots{ }^{A}$ & $47.29( \pm 0.57) \mathrm{a}$ & $-{ }^{B}$ & $22.35( \pm 0.56) \mathrm{a}$ & $-\cdots{ }^{C}$ & $34.37( \pm 0.33)$ a & $-\cdots{ }^{D}$ \\
\hline 2 & $43.99( \pm 2.30) b$ & 8.18 & $45.46( \pm 0.57) \mathrm{b}$ & 3.87 & $23.16( \pm 0.85)$ a & 3.62 & $35.04( \pm 0.25)$ a & 1.94 \\
\hline 3 & $42.78( \pm 2.51) b$ & 10.70 & $43.90( \pm 1.52) \mathrm{c}$ & 7.17 & $23.26( \pm 1.00) \mathrm{a}$ & 4.07 & $35.52( \pm 0.81) a b$ & 3.35 \\
\hline 4 & $41.27( \pm 2.51) b$ & 13.86 & $43.00( \pm 0.57) \mathrm{c}$ & 9.07 & $24.29( \pm 1.07)$ a & 8.68 & $37.49( \pm 2.34) b$ & 9.08 \\
\hline 5 & $4.30( \pm 1.00) \mathrm{C}$ & 91.02 & $3.90( \pm 0.57) d$ & 91.75 & $77.79( \pm 4.63) \mathrm{b}$ & 248.0 & $83.22( \pm 0.73) \mathrm{c}$ & 142.13 \\
\hline
\end{tabular}

Valores corresponden a promedios de tres repeticiones con su respectiva desviación estándar. Letras distintas indican medias estadísticamente diferentes $(P<0.05)$.

${ }^{\mathrm{A}}{ }^{\mathrm{B}} \quad$ Pérdidas (\%) de celulosa en madera de $N$. betuloides y $S$. conspicua respecto al estado inicial de degradación.

${ }^{\mathrm{C}} \mathrm{y}^{\mathrm{D}}$ Incrementos (\%) en la proporción de lignina en $N$. betuloides y S. conspicua respecto al estado inicial de degradación.

\section{DISCUSIÓN}

El volumen (632 $\mathrm{m}^{3} \mathrm{ha}^{-1}$ ) y necromasa (231,5 $\mathrm{Mg} \mathrm{ha}^{-1}$ ) de RLC en descomposición, muestra que el bosque templado de antiguo crecimiento estudiado, posee cantidades significativas de madera muerta, donde las mayores contribuciones las hace $N$. betuloides con el 95,2\%, respecto a $S$. conspicua, L. philippiana y D. diacanthoides. Estos valores pueden considerarse como representativos para bosques templados siempreverdes de antiguo crecimiento del sur de Chile, y demuestran mayores aportes de madera muerta al piso forestal, que los bosques simpreverdes de coníferas del hemisferio norte (Carmona et al., 2002). El volumen de RLC depositados en el bosque, representó el 16,4\% del volumen fustal $\left(2.394,9 \mathrm{~m}^{3} \mathrm{ha}^{-1}\right)$ de los árboles vivos por ha ${ }^{-1}$, reflejando plena dominancia de $N$. betuloides, especie sombra-intolerante, cuyos restos maderables depositados en el suelo, liberan espacios con acceso a luz, que son colonizados por la regeneración natural (Brown et al., 2013).

La mayor parte de RLC depositados en el suelo del bosque (632 $\mathrm{m}^{3}$ $\mathrm{ha}^{-1} \approx 231,5 \mathrm{Mg} \mathrm{ha}^{-1}$ ) se encontraron en los estados más avanzados de descomposición (4 y 5), lo cual indica que el bosque es de crecimiento antiguo, y la madera que actualmente se encuentra en degradación, sería el resultado de disturbios naturales ocurridos hace varias décadas, concordante 
con lo señalado por Lombardi et al. (2011). Bajo estas condiciones, la madera en degradación avanzada, constituye un lugar de intensa actividad microbiológica, principalmente fúngica, que actúan en la transformación y aprovechamiento relativamente rápido de elementos lábiles, (almidones, pectina, proteínas) y luego en la depolimerización gradual de biomoléculas más complejas, como celulosa y lignina (Dill \& Kraepelin, 1986; Blanchette, 1991).

La mayor solubilidad de madera en el estado 5 de descomposición, estaría asociado a la pérdida de sus propiedades estructurales, principalmente por actividad de la biomasa microbiana, donde los hongos ligninocelulolíticos colonizan la madera en cuanto esta llega al suelo y empiezan el proceso de degradación de celulosa, lignina y resinas (Blanchette, 1991; Martínez et al., 2005). Cuanto más degradada está la madera, la concentración de carbohidratos de bajo peso molecular, como pentosanos, hexosanos (poliosas) y celulosas degradadas, aumentan, siendo indicadores robustos de la actividad fúngica (TAPPI, 2000).

Los porcentajes más altos de celulosa se encontraron en madera recién caída al suelo (estado inicial de degradación) de $N$. betuloides (47.91\%) y S. conspicua (47.29\%), disminuyendo gradualmente en función de la degradación, hasta llegar al valor extremo de $4.3 \%$ y $3.9 \%$ en ambas especies forestales, respectivamente, lo cual constituye un excelente indicador del metabolismo microbiano, especialmente de hongos que degradan celulosa para obtener fuentes carbonadas (Watkinson et al., 2006).

El contenido de lignina en la madera, presentó un comportamiento opuesto a la celulosa, donde los mayores porcentajes de este componente orgánico recalcitrante, se encontró en el estado más avanzado de degradación. Los contenidos de lignina en madera de $S$. conspicua fueron levemente superiores a $N$. betuloides, en todos los estados de descomposición, situación que puede ser explicada en que las coníferas por lo general, son más ricas en este componente orgánico, hasta en un 10\% por sobre las latifoliadas (Rodríguez \& Torres, 1994). El contenido inicial de lignina en ambas especies forestales, estuvo dentro de los rangos establecidos para latifoliadas y coníferas (Carmona \& Urzúa, 2013), pero el aumento de su concentración estuvo en función del deterioro de la madera (Agosin et al.1990), que por actividad microbiana lignino-celulolítica, redujo la celulosa a componentes energéticos, quedando en la madera únicamente compuestos recalcitrantes, principalmente lignina (Dill \& Kraepelin 1986, Martínez et al. 2005).

\section{CONCLUSION}

- La mayor cantidad de restos lignino-celulósicos acumulados en el bosque templado siempreverde de antiguo crecimiento, pertenecieron 
a $N$. betuloides (95,23\%), con un almacenamiento de $632 \mathrm{~m}^{3} \mathrm{ha}^{-1}$ que representaron 231,5 $\mathrm{Mg} \mathrm{ha}^{-1}$ de necromasa, cuyos restos se encontraron principalmente en los estados 4 (56,5\%) y $5(34,1 \%)$ de descomposición, indicando que la mayor parte de estos materiales orgánicos, están en degradación avanzada.

- La necromasa de $N$. betuloides y $S$. conspicua mostró mayor solubilidad en el estado de descomposición más avanzado (estado 5), con un incremento del $274 \%$ y $819 \%$ con respecto al estado inicial de degradación, respectivamente.

- Los bajos contenidos de celulosa y altas proporciones de lignina en la madera perteneciente al último estado de descomposición (5), son indicadores que la biomasa microbiana aprovecha el carbono atrapado, y libera de $\mathrm{CO}_{2}$ hacia la atmosfera como producto de su metabolismo.

\section{References:}

AGOSIN, A., BLANCHETTE, R.A., SILVA, H., LAPIERRE, C., CEASE, K.R., IBACH, R.E., ABAD, A.R. \& MUGAS, P. 1990. Characterization of palo podrido, a natural process of delignification in wood. Applied and Environmental Microbiology 56(1): 65-74.

BELEZACA, C., SALVATIERRA, D., DELGADO, D., GODOY, R., VALENZUELA, E., JIMENEZ, E., LOPEZ, R., BAQUE, R., HERRERA, R., OVIEDO, B., CADME, M., BOHORQUEZ, T. \& VÁSCONEZ, C. 2016. Acumulación de restos lignino-celulósicos (necromasa) en un bosque templado de antiguo crecimiento dominado por Nothofagus en el Centro-Sur de Chile. European Scientific Journal 12(9): 69-80.

BLANCHETTE, R.A. 1991. Delignification by wood-decay fungi. Annual Review of Phytopathology 29: 381-398.

BROWN, M.J., KERTIS, J. \& HUFF, M.H. 2013. Natural Tree Regeneration and Coarse Woody Debris Dynamics After a Forest Fire in the Western Cascade Range. USDA, USA. 50 p.

CARMONA, C. \& URZÚA, A. 2013. Caracterización de biomasa leñosa con fines energéticos disponibles en Chile. Universidad de Chile. 134 p.

CARMONA, M.R., ARMESTO, J.J., ARAVENA, J.C. \& PÉREZ, C.A. 2002. Coarse woody debris biomass in successional and primary temperate forests in Chiloé Island, Chile. Forest Ecology and Management, 164(1-3): 265-275.

CÉSARI, S.N., BUSQUETS, P., COLOMBO-PIÑOL, F., MÉNDEZBEDIA, I. \& LIMARINO, C.O. 2010. Nurse logs: An ecological strategy in a late Paleozoic forest from the southern Andean region. Geology, 38(4): 295-298. 
CRESPO, R., TORRES, M., VALENZUELA, L. \& POBLETE, H. 2013. Propiedades químicas, color y humectabilidad de partículas de Laureliopsis philippiana (tepa) con y sin tratamiento térmico. Maderas, Ciencia y Tecnología 15(3): 337-348.

DILL, I. \& KRAEPELIN, G. 1986. Palo Podrido: Model for extensive delignification of Wood by Ganoderma applanatum. Applied and Environmental Microbiology 52(6):1305-1312.

GAYOSO, J. 2001. Medición de la capacidad de captura de carbono en bosques nativos y plantaciones de Chile. Revista Forestal Iberoamericana, 1(1): 1-13.

GONZÁLEZ-POLO, M., FERNÁNDEZ-SOUTO, A. \& AUSTIN, A.T. 2013. Coarse Woody Debris Stimulates Soil Enzymatic Activity and Litter Decomposition in an Old-Growth Temperate Forest of Patagonia, Argentina. Ecosystems, 16(6): 1025-1038.

HEINEMANN, K. \& KITZBERGER, T. 2006. Effects of position, understory vegetation and coarse woody debris on tree regeneration in two environmentally contrasting forests of north-western Patagonia: a manipulative approach. Journal of Biogeography, 33(8): 1357-1367.

JIA-BING, W., DE-XIN, G., SHI-JIE, H., MI, Z. \& CHANG-JIE, J. 2005. Ecological functions of coarse woody debris in forest ecosystem. Journal of Forestry Research, 16(3): 247-252.

LOMBARDI, F., COCOZZA, C., LASSERRE, B., TOGNETTI, R., \& MARCHETTI, M. 2011. Dendrochronological assessment of the time since death of dead wood in an old growth Magellan's beech forest, Navarino Island (Chile). Austral Ecology, 36(3): 329-340.

MARRA, J.L. \& EDMONDS, R.L. 1994. Coarse woody debris and forest floor respiration in an old-growth coniferous forest on the Olympic Peninsula, Washington, USA. Canadian Journal of Forest Research, 24(9): 1811-1817.

MARTÍNEZ, A.T., SPERANZA, M., RUIZ-DUEÑAS, F.J., FERREIRA, P., CAMARERO, S., GUILLEN, F., MARTÍNEZ, M.J., GUTIERREZ, A. \& DEL RÍO, J.C. 2005. Biodegradation of lignocellulosics: microbial, chemical, and enzymatic aspects of the fungal attack of lignin. International Microbiology 8(3): 195-204.

OYARZÚN, C.E., GODOY, R., SCHRIJVER, A., STAELENS, J. \& LUST, N. 2004. Water chemistry and nutrient budgets in an undisturbed evergreen rainforest of southern Chile. Biogeochemistry, 71: 107-123.

RODRÍGUEZ, S. \& TORRES, M. 1994. Análisis cuantitativo de componentes principales y secundarios en la corteza de diversas especies nativas y exóticas de la Provincia de Llanquihue, X Región, Chile. Bosque 15(2): 45-48. 
SCHLEGEL, B.C. \& DONOSO, P.J. 2008. Effects of forest type and stand structure on coarse woody debris in Oldgrowth rainforests in the Valdivian Andes, south-central Chile. Forest Ecology and Management, 255: 19061914.

SOIL SURVEY STAFF. 1999. Soil Taxonomy. A basic system of soil classification for making and interpreting soil surveys, 2nd edition. Agricultural Handbook 436, Natural Resources Conservation Service, USDA, Washington DC, USA, pp. 869.

SPIES, T.A., FRANKLIN, J.F. \& THOMAS, T.B. 1988. Coarse woody debris in Douglas-fir forest of western Oregon and Washington. Ecology, 69: 1689-1702.

TAPPI. Technical Assotiation of the Pulp and Paper Industry. 2000. TAPPI Test Methods 2000 - 2001. TAPPI Press. Atlanta, United States.

WATKINSON, S.C., BEBBER, D., DARRAH, P.R., FRICKER, M.D., TLALKA, M. \& BODDY, L. 2006. The role of wood decay fungi in the carbon and nitrogen dynamics of the forest floor. In Fungi in biogeochemical cycles. GM Gadd (Eds). Cambridge University Press. Cambridge, UK. pp. 151-181.

YAN, E., WANG, X. \& HUANG, J. 2006. Concept and Classification of Coarse Woody Debris in Forest Ecosystems. Frontiers of Biology in China, 1(1):76-84. 